\title{
A Sauer-Shelah-Perles Lemma for Sumsets
}

\author{
Zeev Dvir* \\ Department of Computer Science and Department of Mathematics \\ Princeton University \\ Princeton, U.S.A. \\ zeev.dvir@gmail.com \\ Shay Moran $^{\dagger}$ \\ School of Mathematics \\ Institute for Advanced Study \\ Princeton, U.S.A. \\ shaymoran1@gmail.com
}

Submitted: Jun 20, 2018; Accepted: Oct 23, 2018; Published: Nov 16, 2018

(C) Zeev Dvir and Shay Moran. Released under the CC BY-ND license (International 4.0).

\begin{abstract}
We show that any family of subsets $A \subseteq 2^{[n]}$ satisfies $|A| \leqslant O\left(n^{\lceil d / 2\rceil}\right)$, where $d$ is the VC dimension of $\{S \triangle T \mid S, T \in A\}$, and $\triangle$ is the symmetric difference operator. We also observe that replacing $\triangle$ by either $\cup$ or $\cap$ fails to satisfy an analogous statement. Our proof is based on the polynomial method; specifically, on an argument due to [Croot, Lev, Pach '17].
\end{abstract}

Mathematics Subject Classifications: 05D05, 05E99

\section{Introduction}

Let $A \subset 2^{[n]}$ be a family of subsets of an $n$ element set ([n] w.l.o.g). The VC dimension of $A$, denoted by $\mathrm{VC}-\operatorname{dim}(A)$, is the size of the largest $Y \subseteq[n]$ such that $\{S \cap Y \mid S \in A\}=2^{Y}$. One of the most useful facts about the VC dimension is given by the Sauer-Shelah-Perles Lemma.

Theorem 1 (Sauer-Shelah-Perles Lemma $[12,13])$. Let $d \leqslant n \in \mathbb{N}$. Suppose $A \subset 2^{[n]}$ satisfies $\operatorname{VC}-\operatorname{dim}(A) \leqslant d$. Then $|A| \leqslant\left(\begin{array}{c}n \\ \leqslant d\end{array}\right)$.

\footnotetext{
*Research supported by NSF CAREER award DMS-1451191 and NSF grant CCF-1523816.

${ }^{\dagger}$ Research supported by the National Science Foundation under agreement No. CCF-1412958 and by the Simons Foundations.
} 
The Sauer-Shelah-Perles Lemma has numerous applications ranging from model theory, probability theory, geometry, combinatorics, and various fields in computer science. A simple-yet-useful corollary of this lemma is that if $\mathrm{VC}-\operatorname{dim}(A) \leqslant d$, and $\star$ is any binary set-operation (e.g. $\star \in\{\cap, \cup, \triangle\}$ ) then

$$
|\{S \star T \mid S, T \in A\}| \leqslant\left(\begin{array}{c}
n \\
\leqslant d
\end{array}\right) \cdot\left(\begin{array}{c}
n \\
\leqslant d
\end{array}\right)=O\left(n^{2 d}\right) .
$$

This corollary is used, for example, by [2] to derive closure properties for PAC learnability. Let $A \circledast A$ denote the family $\{S \star T \mid S, T \in A\}$. In this work we explore the converse direction: Does an upper bound on the VC-dimension VC-dim $(A \circledast A)$ imply an upper bound on $|A|$ ? It is not hard to see that VC-dim $(A) \leqslant \operatorname{VC}-\operatorname{dim}(A \circledast A)$ for $\star \in\{\cup, \cap, \triangle\}$, and therefore, by Theorem 1: VC- $\operatorname{dim}(A \circledast A)<d \Longrightarrow|A| \leqslant O\left(n^{d}\right)$.

Our main result quadratically improves this naive bound when $\star$ is symmetric difference:

Theorem 2. Let $d \leqslant n \in \mathbb{N}$. Suppose $A \subset 2^{[n]}$ satisfies VC-dim $(A \triangle A) \leqslant d$. Then

$$
|A| \leqslant 2\left(\begin{array}{c}
n \\
\leqslant\lfloor d / 2\rfloor
\end{array}\right)
$$

We note that Theorem 2 does not hold when $\star \in\{\cup, \cap\}$ : pick $d \geqslant 2$, and set

$$
A=\{S \subseteq[n]|| S \mid \leqslant d\}
$$

Note that $A=A \cap A$ and therefore $d=\operatorname{VC}-\operatorname{dim}(A)=\operatorname{VC}-\operatorname{dim}(A \cap A)$. However $|A|=$ $\left(\begin{array}{c}n \\ \leqslant d\end{array}\right)=\Theta\left(n^{d}\right)$, which is not upper bounded by $O\left(n^{\lceil d / 2\rceil}\right)$. Picking $A=\{S \subseteq[n]|| S \mid \geqslant$ $n-d\}$ shows that $\cup$ behaves similarly to $\cap$ in this context.

The above examples rule out the analog of Theorem 2 for exactly one of $\cup, \cap$. This suggests the following open question:

Question 3. Let $d \leqslant n \in \mathbb{N}$. Suppose $A \subset 2^{[n]}$ satisfies VC-dim $(A \cap A) \leqslant d$ and VC- $\operatorname{dim}(A(\cup) A) \leqslant d$. Is it necessarily the case that $|A| \leqslant n^{d / 2+O(1)}$ ?

Another natural question is whether this phenomenon extends to several applications of the symmetric difference operator, for example:

Question 4. Does there exist an $\epsilon<1 / 2$ such that for every $d \leqslant n$ and every $A \subset 2^{[n]}$ :

$$
\operatorname{VC}-\operatorname{dim}(A \triangle A \triangle A) \leqslant d \Longrightarrow|A| \leqslant n^{\epsilon \cdot d+O(1)} ?
$$

In Section 3 we derive a related statement when $\triangle$ is replaced by addition modulo $p$ for a prime $p$, and the $\mathrm{VC}$ dimension is replaced by the interpolation degree (which is defined in the next section). 


\subsection{Interpolation degree}

Since our proof method is algebraic, it is convenient to view $A \subset 2^{[n]}$ as a subset of the $n$-dimensional vector space $\mathbb{F}_{2}^{n}$ over the field of two elements. In this setting $A \triangle A$ is the sumset of $A$, denoted $A+A$.

Theorem 2 will follow from a stronger statement involving a quantity referred to in some places as the regularity (as a special case of Castelnuovo-Mumford regularity from algebraic geometry) [11] and in others as the interpolation-degree [9]. We will use the more descriptive interpolation-degree for the rest of this paper. We begin with some preliminary notations and definitions.

Let $A \subset \mathbb{F}_{2}^{n}$. It is a basic fact that for each function $f: \mathbb{F}_{2}^{n} \mapsto \mathbb{F}_{2}$ there exists a unique multilinear polynomial $P_{f} \in \mathbb{F}_{2}\left[x_{1}, \ldots, x_{n}\right]$ such that $f(a)=P_{f}(a)$ for all $a \in \mathbb{F}_{2}^{n}$ (existence is via simple interpolation and uniqueness follows from dimension counting). For a partial function $f: A \mapsto \mathbb{F}_{2}$ there are many (precisely $2^{2^{n}-|A|}$ ) multilinear polynomials whose restriction to $A$ computes $f$. Let $\operatorname{deg}_{A}(f)$ denote the minimal degree of any polynomial whose restriction to $A$ computes $f$. We define the interpolation-degree of $A$, denoted int-deg $(A)$ to be the maximum of $\operatorname{deg}_{A}(f)$ taken over all functions $f: A \mapsto \mathbb{F}_{2}$. In other words, int-deg $(A)$ is the smallest $d$ such that any function from $A$ to $\mathbb{F}_{2}$ can be realized by a polynomial of degree at most $d$. Clearly, int-deg $(A)$ is an integer between 0 and $n$. It is also not hard to see that, if $A$ is a proper subset of $\mathbb{F}_{2}^{n}$ then int-deg $(A)<n$. Our interest in int-deg $(A)$ comes from the following connection to VC-dimension.

Lemma $5([1,8,14,9])$. For $A \subset \mathbb{F}_{2}^{n}$ we have int-deg $(A) \leqslant \operatorname{VC}$-dim $(A)$.

This Lemma, under various formulations, was proved in several works. The formulation that appears here can be found in [9]. For completeness, we next sketch the proof: since the set of all multilinear monomials (also those of degree larger than $\mathrm{VC}-\operatorname{dim}(A)$ ) span the set of functions $f: A \rightarrow \mathbb{F}_{2}$, it suffices to show that any monomial (when seen as an $A \rightarrow \mathbb{F}_{2}$ function) can be represented a polynomial of degree at most $d=\operatorname{VC}-\operatorname{dim}(A)$. The crucial observation is that if $x_{S}=\pi_{i \in S} x_{i}$ is a monomial of degree larger than $d$, then $S$ is not shattered by $A$. This means that there is a pattern $v: S \rightarrow\{0,1\}$ that does not appear in any of the vectors in $A$ and therefore

$$
\Pi_{i \in S}\left(x_{i}+v_{i}+1\right)={ }_{A} 0,
$$

where " $={ }_{A} "$ means equality as functions over $A$. Now, expanding this product and rearranging the equation yields a representation of $x_{S}$ as sum of monomials $x_{S^{\prime}}$, where $S^{\prime} \subset S$, which by induction can also be represented by polynomials of degree at most $d$.

Theorem 5 reduces Theorem 2 to the following stronger statement that is proved in the next section.

Theorem 6. Let $d \leqslant n \in \mathbb{N}$, and let $A \subset \mathbb{F}_{2}^{n}$ satisfy $|A|>2\left(\begin{array}{c}n \\ \leqslant\lfloor d / 2\rfloor\end{array}\right)$. Then int-deg $(A+$ A) $>d$. 


\section{Proof of Theorem 6}

The main technical tool will be a lemma of Croot-Lev-Pach [3] that was the main ingredient in the recent solution of the cap-set problem [5] and has found many other applications since then (e.g., $[7,15,4,6]$ to name a few).

Lemma 7 (CLP lemma [3]). Let $P \in \mathbb{F}_{q}\left[x_{1}, \ldots, x_{n}\right]$ be a polynomial of degree at most $d$ over any finite field $\mathbb{F}_{q}$, and let $M$ denote the $q^{n} \times q^{n}$ matrix with entries $M_{x, y}=P(x+y)$ for $x, y \in \mathbb{F}_{q}^{n}$. Then $\operatorname{rank}(M) \leqslant 2 \cdot m_{\lfloor d / 2\rfloor}(q, n)$, where $m_{k}(q, n)$ denotes the number of monomials in $n$ variables $x_{1}, \ldots, x_{n}$ such that each variable appears with individual degree at most $q-1$ and the total degree of the monomial is at most $k$.

Specializing to our setting of $\mathbb{F}_{2}$ multilinear polynomials, we see that $m_{k}(2, n)=\left(\begin{array}{c}n \\ \leqslant k\end{array}\right)$ and so we conclude:

Corollary 8. Let $P \in \mathbb{F}_{2}\left[x_{1}, \ldots, x_{n}\right]$ be a polynomial of degree at most $d$ and let $M$ be as in Lemma 7. Then $\operatorname{rank}(M) \leqslant 2\left(\begin{array}{c}n \\ \leqslant\lfloor d / 2\rfloor\end{array}\right)$.

We are now ready to prove Theorem 6 .

Proof of Theorem 6. Suppose $A \subset \mathbb{F}_{2}^{n}$ is such that $|A| \geqslant 2\left(\begin{array}{c}n \\ \leqslant\lfloor d / 2\rfloor\end{array}\right)$. Let $f: A+A \mapsto \mathbb{F}_{2}$ be such that $f(\overline{0})=1$, where $\overline{0}$ is the all zero vector in $\mathbb{F}_{2}^{n}$, and $f(a)=0$ for all non-zero $a \in A+A$. It suffices to show that $\operatorname{deg}_{A+A}(f) \geqslant\lfloor d / 2\rfloor$ (notice that since $A \neq \emptyset$ it follows that $\overline{0} \in A+A$ and so $f$ is not constantly 0 on $A+A$ ). Let $M$ be the $2^{n} \times 2^{n}$ matrix whose rows and columns are indexed by $\mathbb{F}_{2}^{n}$ and with entries $M_{x, y}=f(x+y)$. By our definition of $f$ we have that the sub-matrix of $M$ whose rows and columns are indexed by $A$ is just the $|A| \times|A|$ identity matrix. This implies

$$
\operatorname{rank}(M) \geqslant|A|
$$

Let $d_{f}=\operatorname{deg}_{A+A}(f)$ denote the smallest degree of a polynomial whose restriction to $A+A$ computes $f$. Applying Corollary 8 we get that

$$
\operatorname{rank}(M) \leqslant 2\left(\begin{array}{c}
n \\
\leqslant\left\lfloor d_{f} / 2\right\rfloor
\end{array}\right)
$$

Combining the two inequalities on $\operatorname{rank}(M)$ and using the bound on the size of $A$ we get that

$$
2\left(\begin{array}{c}
n \\
\leqslant\lfloor d / 2\rfloor
\end{array}\right)<|A| \leqslant \operatorname{rank}(M) \leqslant 2\left(\begin{array}{c}
n \\
\leqslant\left\lfloor d_{f} / 2\right\rfloor
\end{array}\right),
$$

which implies $\lfloor d / 2\rfloor<\left\lfloor d_{f} / 2\right\rfloor$. This means that $d_{f}>d$ and so int-deg $(A+A)>d$. 


\section{Generalization to sums modulo $p$}

In this section we observe that our proof can be generalized to give stronger bounds in the case when we take $p$-fold sums of boolean vectors over $\mathbb{F}_{p}$. The case proved in the last section corresponds to (two fold) sums modulo 2. For a subset $A \subset \mathbb{F}_{p}^{n}$ and a positive integer $k$, we denote by

$$
k \cdot A=\left\{a_{1}+\ldots+a_{k} \mid a_{i} \in A\right\}
$$

the $k$-fold sumset of $A$. To formally define the interpolation degree over $\mathbb{F}_{p}$ we need to consider, instead of multilinear polynomials, polynomials in which each variable has degree at most $p-1$. We call such polynomials $p$-reduced polynomials. The space of all $p$-reduced polynomials has dimension $p^{n}$ and can uniquely represent any function $f: \mathbb{F}_{p}^{n} \mapsto \mathbb{F}_{p}$. The degree of such a function is defined to be the total degree of the unique $p$-reduced polynomial representing it and can range between 0 and $(p-1) n$. The interpolation degree of a set $A \subset \mathbb{F}_{q}^{n}$ is the minimum $d$ such that any function $f: A \mapsto \mathbb{F}_{p}$ can be represented by a $p$-reduced polynomial of degree at most $d$. To avoid confusion we will denote the interpolation degree over $\mathbb{F}_{p}^{n}$ as int-deg $(A)$.

We denote by $\mathcal{M}_{d}(p, n)$ the set of monomials in $n$ variables $x_{1}, \ldots, x_{n}$ in which each variable has degree at most $p-1$ and the total degree is at most $d$. When $p=2$ we have the closed formula $\left|\mathcal{M}_{d}(2, n)\right|=\left(\begin{array}{c}n \\ \leqslant d\end{array}\right)$. When $p>2$ the quantity $\left|\mathcal{M}_{d}(p, n)\right|$ is a bit more tricky to compute but is known to satisfy certain asymptotic inequalities (e.g., large deviations [10] showing that $\mathcal{M}_{\delta n}(p, n) \leqslant 2^{\epsilon n}$ with $\epsilon(\delta)$ going to zero with $\delta$ ).

The following theorem generalizes Theorem 2 when $p>2$.

Theorem 9. Let $p$ be any prime number and let $A \subset\{0,1\}^{n} \subset \mathbb{F}_{p}^{n}$ be such that $|A|>$ $p \cdot\left|\mathcal{M}_{\lfloor d / p\rfloor}(p, n)\right|$. Then int-deg $p(p \cdot A)>d$.

The proof of the theorem requires the notion of slice-rank of a tensor which was introduced by Tao in his symmetric interpretation of the proof of the cap-set conjecture [16]. By a $k$-fold tensor of dimension $D$ over a field $\mathbb{F}$ we mean a function $T$ mapping ordered tuples $\left(j_{1}, \ldots, j_{k}\right) \in[D]^{n}$ to $\mathbb{F}$. The slice-rank of a $k$-fold tensor $T$ is a the smallest integer $R$ such that $T$ can be written as a sum $T=\sum_{i=1}^{R} T_{i}$ such that, for every $i \in[R]$ there is some $j_{i} \in[k]$ so that $T_{i}\left(j_{1}, \ldots, j_{k}\right)=A\left(j_{i}\right) B\left(j_{1}, \ldots, j_{i-1}, j_{i+1}, \ldots, j_{k}\right)$. In other words, we define the 'rank one' tensors to be those in which the dependence on one of the variables is multiplicative (by a function $A\left(j_{i}\right)$ ) and the rank of a tensor is the smallest number of rank one tensors needed to describe it. For 2-fold tensors (or matrices) this notion coincides with the usual definition of matrix rank.

The proof of Theorem 9 will follow from a combination of two lemmas regarding slice rank. The first lemma generalizes the Croot-Lev-Pach lemma (and proved in an a similar way).

Lemma 10. Let $f: \mathbb{F}_{p}^{n} \mapsto \mathbb{F}_{p}$ be of degree $d$. Then the $p$-fold $p^{n}$ dimensional tensor $T:\left(\mathbb{F}_{p}^{n}\right)^{k} \mapsto \mathbb{F}_{p}$ defined by $T\left(X^{1}, \ldots, X^{p}\right)=f\left(X^{1}+\ldots+X^{p}\right)$ has slice rank at most $p \cdot \mathcal{M}_{\lfloor d / p\rfloor}(p, n)$. 
Proof. Consider $T$ as a polynomial in $p$ groups of variables $X^{i}=\left(x_{1}^{i}, \ldots, x_{n}^{i}\right)$ with $i=$ $1,2, \ldots, p$. Since the degree of $f$ is $d$, the degree of $T$ as a polynomial will also be at most $d$. This means that, in each monomial of $T\left(X^{1}, \ldots, X^{p}\right)=f\left(X^{1}+\ldots+X^{p}\right)$, the degree of at least one group of variables will be at most $\lfloor d / p\rfloor$. Grouping together monomials according to which group has low degree (if there is more than one group take the one with lowest index) we can represent $T$ as a sum of $p$ tensors, each having rank at most $\mathcal{M}_{\lfloor d / p\rfloor}(p, n)$. This completes the proof.

The second lemma needed to prove Theorem 9 is due to Tao and shows that the 'diagonal' tensor has full rank.

Lemma $11([16])$. Let $\delta\left(j_{1}, \ldots, j_{k}\right):[D]^{k} \mapsto \mathbb{F}$ be defined as $\delta(j, j, \ldots, j)=1$ for all $j$ and is zero otherwise. Then the slice rank of $\delta$ is equal to $D$.

Proof of Theorem 9. To prove the bound on int- $\operatorname{deg}_{p}(p \cdot A)$ we describe a function $f$ : $p \cdot A \mapsto \mathbb{F}_{p}$ that cannot be represented by a low degree polynomial. We take $f$ to be equal to 1 on the zero vector and zero otherwise. We now consider the tensor $T\left(X^{1}, \ldots, X^{p}\right)=$ $f\left(X^{1}+\ldots+X^{p}\right)$ defined on $A^{p}$. Notice that, since $A \subset\{0,1\}^{n}$, the sum of $p$ of them is equal to zero iff all $p$ summands are identical. This implies that $T$ is the diagonal tensor $\delta$ of Lemma 11 and hence has rank equal to $|A|$. On the other hand, if the degree of $f$ (over $p \cdot A)$ is at most $d$ then, by Lemma 10 , the tensor $T$ has rank at most $p \cdot \mathcal{M}_{\lfloor d / p\rfloor}(p, n)$. Since we assume that $|A|>p \cdot \mathcal{M}_{\lfloor d / p\rfloor}(p, n)$ this cannot happen and so int- $\operatorname{deg}_{p}(p A)>d$.

\section{Acknowledgements}

We thank Stijn Cambie for helpful comments on an earlier draft of this manuscript.

\section{References}

[1] L. Babai and P. Frankl. Linear Algebra Methods in Combinatorics. University of Chicago, 1992.

[2] A. Blumer, A. Ehrenfeucht, D. Haussler, and M. K. Warmuth. Learnability and the Vapnik-Chervonenkis dimension. J. Assoc. Comput. Mach., 36(4):929-965, 1989. doi:10.1145/76359.76371.

[3] E. Croot, V. F. Lev, and P. P. Pach. Progression-free sets in $\mathbb{Z}_{4}^{n}$ are exponentially small. Ann. of Math. (2), 185(1):331-337, 2017. doi:10.4007/annals.2017.185.1.7.

[4] Z. Dvir and B. Edelman. Matrix rigidity and the Croot-Lev-Pach lemma. Manuscript, 082017.

[5] J. S. Ellenberg and D. Gijswijt. On large subsets of $\mathbb{F}_{q}^{n}$ with no threeterm arithmetic progression. Ann. of Math. (2), 185(1):339-343, 2017. doi:10.4007/annals.2017.185.1.8. 
[6] J. Fox and L. M. Lovász. A tight bound for green's arithmetic triangle removal lemma in vector spaces. In Proceedings of the Twenty-Eighth Annual ACM-SIAM Symposium on Discrete Algorithms, SODA '17, pages 1612-1617, Philadelphia, PA, USA, 2017. Society for Industrial and Applied Mathematics. URL http://dl . acm . org/citation. cfm?id=3039686. 3039792.

[7] B. Green. Sarkozy's theorem in function fields. Quarterly Journal of Mathematics, 68, 2016.

[8] L. Gurvits. Linear algebraic proofs of VC-dimension based inequalities. In Computational Learning Theory, pages 238-250. Springer, 1997.

[9] S. Moran and C. Rashtchian. Shattered sets and the hilbert function. In MFCS, volume 58 of LIPIcs, pages 70:1-70:14. Schloss Dagstuhl - Leibniz-Zentrum fuer Informatik, 2016.

[10] F. Rassoul-Agha and T. Seppäläinen. A course on large deviations with an introduction to Gibbs measures. American Mathematical Society, 05 2015. ISBN 978-0-82187578-0.

[11] Z. Remscrim. The hilbert function, algebraic extractors, and recursive fourier sampling. In 2016 IEEE 57th Annual Symposium on Foundations of Computer Science (FOCS), pages 197-208, Oct 2016. doi:10.1109/FOCS.2016.29.

[12] N. Sauer. On the density of families of sets. J. Comb. Theory, Ser. A, 13:145-147, 1972. doi:10.1016/0097-3165(72)90019-2.

[13] S. Shelah. A combinatorial problem; stability and order for models and theories in infinitary languages. Pacific J. Math., 41(1):247-261, 1972.

[14] R. Smolensky. Well-known bound for the VC-dimension made easy. Computational Complexity, 6(4):299-300, 1997. doi:10.1007/BF01270383. URL http://dx.doi. org/10.1007/BF01270383.

[15] J. Solymosi. The sum of nonsingular matrices is often nonsingular. Linear Algebra and its Applications, 552, 012018.

[16] T. Tao. A symmetric formulation of the Croot - Lev - Pach - Ellenberg - Gijswijt capset bound. https://terrytao.wordpress.com/2016/05/18/ a-symmetric-formulation-of-the-croot-lev-pach-ellenberg, 2016. 